\title{
Bt-Horizonte als Interglazial-Zeiger in den humiden Mittelbreiten: Bildung, Mikromorphologie, Kriterien
}

\author{
SIEGFRIED STEPHAN*)
}

STEPHAN, S. (2000): Bt-Horizonte als Interglazial-Zeiger in den humiden Mittelbreiten: Bildung, Mikromorphologie, Kriterien. - Eiszeitalter und Gegenwart, 50: 95-106; Hannover 2000.

Keywords: paleosol, Bt horizon, interglacial, micropedology.

Kurzfassung: Die Untersuchung wird auf die humiden Mittelbreiten begrenzt, wo „Interglazial“ durch die Rückwanderung temperierter Laubwälder definierbar ist. Auf die Frage, welche Bodentypen oder Horizonte sicher einem Interglazial zugeordnet werden können, gibt es keine eindeutige Antwort. Pedogenetische Betrachtungen und mikromorphologische Befunde führen aber zu einer Fallunterscheidung, die weitreichende Interpretationssicherheit gibt. Sichere Zeugen für eine interglaziäre Genese sind zunächst autochthone Bt-Horizonte aus kalkhaltigen, schluffreichen Substraten und mit Tonbelägen in Poren. Aber auch Bt-Horizonte aus allochthonem Bodenmaterial kalkhaltiger, schluffreicher Substrate und mit Tonbelägen oder deren Bruchstücken sind sichere Zeugerr, wenn die Zeitspanne von Verwitterung, Lessivierung und Materialverlagerung zusammengefasst werden kann und keine Kaltzeit enthält.

Bei anderen Bt-Horizonten ist die Zugehörigkeit zu einem Interglazial sehr zurückhaltend zu beurteilen. Es muss dann sichergestellt werden, dass die Entwicklungszeit nicht durch eine oder mehrere der folgenden Bedingungen so weit verkürzt worden ist, dass kein Interglazial notwendig wäre: 1. Armes Ausgangsmaterial mit geringer Menge verwitterbarer Minerale, 2. Dominanz von Sand und Kies, 3. Beimengung von vorverwittertem und insbesondere Bt-Material aus einem älteren Interglazial, 4. Durchgriff der Verwitterung und Lessivierung aus einem hangenden Boden, 5. Siedlungslage mit Asche-Einfluss.

\section{[Bt-horizons as indicators for interglacial conditions in the humide middle latitudes: genesis, micromorphology, criterions]}

Abstract: The investigation is restricted to the humide middle latitudes, where "interglacial" is definable by the return of temperate deciduous woods. There is no definite answer to the question, what type of soil or horizon may be strongly related to interglacial forming-conditions. However, pedogenetical studies and micromorphological facts lead to a discrimination of situations, giving enough security for the intended interpre-

*) Anschrift des Verfassers: Dr. S. Stephan, Weidenfeld 5, D-53359 Rheinbach; Stephan-Rheinbach@t-online.de tation. Reliable signs of interglacial forming are, first of all, autochthoneous horizons with clay illuviation ( $\mathrm{Bt}$ horizons), if formed from calcareous, silty material, and showing clay cutans in pores. However, Bt horizons formed from allochthoneous soil sediments of such materials are also reliable signs, if there was no glacial phase between weathering, clay illuviation, and transport.

Bt horizons which do not meet these requirements, have to be strongly judged before taken as a criterion: It must be verified, that their evolution time was not shortened by one or more of the following situations in such a way, that interglacial conditions are unnecessary: 1. Poor original material with few weatherable minerals, 2. predominance of sand and gravel, 3. admixture of weathered material of some older interglacial time, in particular Bt material, 4. passage of weathering and lessivation from a soil above through some intermedium, 5. cultivated site with influence of ash.

\section{Einleitung}

Interglaziale sind Phasen des Quartärs, in denen in den humiden Mittelbreiten die Einwanderung temperierter Laubwälder gelungen ist, weil Klima und Boden die Existenz der entsprechenden Baumarten erlaubt haben und die Zeit für ihre Rückwanderung aus den eiszeitlichen Refugien ausgereicht hat. Entsprechende abiotische Bedingungen können auch in kürzeren Erwärmungszeiten, den Interstadialen, erfüllt gewesen sein, wobei sich aber noch keine temperierten Laubwälder etablieren konnten. Eine allgemeinere Definition der Interglaziale mit den Mitteln der vergleichenden Bodenkunde entsprechend den Untersuchungen von BRONGER (z. B. 1999) wird hier nicht vorgenommen.

Paläoböden sind für die Gliederung des Quartärs interessant, weil sie Phasen geomorphologischer Stabilität mit der Möglichkeit zur Bodenbildung markieren. Es wäre besonders wichtig, die Interglaziale an ihren Böden zu erkennen; aber die bodenkundlichen Kriterien für Interglazialbedingungen sind umstritten. Schon 1968 erchien eine zusammenfassende Arbeit zur Frage „Spätglazial oder Holozän?" (RoHdenburg \& Meyer, 1968); doch die Diskussion, die in verstreuten Publikationen geführt wird, ist noch nicht beendet. Einig- 
keit scheint darin zu bestehen, dass von den quartären Bodenhorizonten nur Bt-Horizonte, also Bodenhorizonte mit Einwaschung von reinem, in Poren nachweisbarem Ton, und damit von den Böden nur die Lessivés an Interglaziale gebunden sein können; aber es muss geklärt werden, wann diese Bindung zwingend ist.

Dies ist für die Paläoböden in mächtigen Lössprofilen weitgehend der Fall, kann aber sicher nicht verallgemeinert werden; denn erstens sind auch aus den Spätweichsel-Interstadialen Bt-Horizonte bekannt, und zweitens wurde gezeigt, dass in einigen Holozän-Profilen die Lessivierung so schnell abgelaufen ist, dass diese ebenso in einem Interstadial zum Ziele gekommen wäre.

Dieser Beitrag befasst sich mit der Suche nach differenzierenden Merkmalen, die solche Bt-Horizonte, für deren Bildung ein Interglazial notwendig ist, von anderen trennen, die kein Interglazial benötigen. Gesucht wird also nach Bt-Horizonten als hinreichende Interglazial-Kriterien, während Bodenhorizonte als notwendige Interglazial-Kriterien, solche also, die einem Interglazialboden nicht fehlen können, selbstverständlich nicht allgemein postuliert werden können (wohl mit den Mitteln der vergleichenden Paläobodenkunde entsprechende Verwitterungsintensitäten, vgl. Bronger 1999).

In Frage kommen Unterschiede in Kalkgehalt und Korngrößenzusammensetzung der Substrate, Merkmale der Bodenumlagerung und wenige andere Kriterien. Für diese Merkmale wird nachfolgend vor allem der Einfluss auf die Entwicklungsdauer untersucht, und zwar im Rahmen einer Darstellung der Prozessfolge, die vom Ausgangssubstrat zum Bt-Horizont führt. Die Kenntnis dieser Prozesse stützt sich in wesentlichen Details auf die Mikromorphologie. Es wird hier nicht versucht, die Prozesse in den holozänen Klima- und Vegetationswandel einzugliedern, obwohl einige Autoren festgestellt haben, dass dieser Änderungen in der Bodenentwicklung verursacht hat (Reuter, 1964).

Die vorliegende Beurteilung gilt nur für Substrate, deren Tonsubstanz von Dreischicht-Tonmineralen dominiert wird. Bei den pleistozänen Substraten Mitteleuropas ist dies der Fall, wenn sie nicht von präpleistozänen Verwitterungsprodukten abstammen. Zweischicht-Tonminerale verhalten sich vor allem deshalb anders, weil sie wegen geringer Schichtladung kaum von mehrwertigen Kationen stabilisiert werden.

\section{Untersuchungs- und Darstellungs- methode aus der Mikromorphologie}

Für die Mikromorphologie werden 2,5 cm x $4 \mathrm{~cm}$ x $20 \mu \mathrm{m}$ bis $5 \mathrm{~cm}$ x $7 \mathrm{~cm}$ x $20 \mu \mathrm{m}$ große Dünnschliffe ungestörter, mit Vestopal imprägnierter Proben im Durchlicht und meist zwischen teilweise gekreuzten Polarisatoren untersucht. Für die Deutung der Profile werden vorwiegend folgende mikroskopische Merkmale herangezogen (STEPHAn 1994): Anteil primärer Grobporen, Art und Verteilung der Carbonate, Anwesenheit von Glimmerplättchen, doppelbrechende Tonlinsen von wenigen $\mu \mathrm{m}$ Größe und Eisenoxidkörnchen und -flocken als Produkte der Glimmerverwitterung, zusammengeflossener doppelbrechender Ton in der Bodenmatrix und Tonbeläge in Poren und Rissen als Produkte der Tonverlagerung, Tonbelags-Fragmente in der Bodenmatrix zum Nachweis transportierten Bt-Materials sowie Eisenkonzentration und Bleichflecken als Zeichen der Redoxprozesse.

Die Abbildungen illustrieren das hier zu Grunde gelegte Konzept der Lessivierung, das von zahlreichen entsprechenden Bodendünnschliffen abstrahiert worden ist. Das Konzept kann damit freilich nicht bewiesen werden, denn die Bilder gehören zu sehr unterschiedlichen Bodenprofilen. Dieser Mangel lässt sich prinzipiell nicht beheben, weil zeitlich aufeinander folgende Entwicklungsphasen solcher Dauer nicht zugleich am gleichen Material unter gleichen Umweltbedingungen untersucht werden können.

\section{Prozessfolge der Verwitterung und Lessivierung}

\subsection{Kalklösung}

Der Zeitbedarf der Kalklösung ist für die Gesamtdauer der Bodenentwicklung wichtig, denn die nachfolgenden Entwicklungsschritte setzen eine vollständige Entkalkung des Bodens voraus. Diese wird vorwiegend durch Perkolation von $\mathrm{CO}_{2}^{-}$ haltigem Wasser bewirkt. In Kaltzeiten ist wegen geringen Kohlendioxidgehaltes der Atmosphäre (Graedel \& KRUTZEN 1994: 4, 242) und geringer $\mathrm{CO}_{2}$-Produktion durch Bodenlebewesen und Wurzeln trotz höherer $\mathrm{CO}_{2}$-Löslichkeit im Wasser nicht mit einer relevanten Entkalkung zu rechnen, die daher weitgehend an Warmzeiten - Interglaziale wie Interstadiale - gebunden zu sein scheint. Die Tschernoseme, die sich in trockenen Lössgebieten (auch in Teilen der Kölner Bucht, Kopp 1965) mindestens bis zum Atlantikum entwickelt hatten, sind sogar erst seit dem Atlanti- 
kum entkalkt worden und in ihren trockensten Arealen oder unter dem Einfluss kalkhaltigen Grundwassers überhaupt erhalten geblieben. Geringer Kalkgehalt im Substrat verkürzt die Entkalkungsphase.

Die Carbonate sind mikroskopisch gut zu erkennen und pedogener von lithogenem Kalk zu unterscheiden (vgl. STtePHAN 1995). Dagegen ist es schwierig, vorhandene Tonsubstanz und deren Orientierungsgrad zu erkennen, wenn Scharen feiner Kalkkristalle das Bild bestimmen.

\subsection{Silicatverwitterung}

Zwar können die spaltbaren Silicate, insbesondere dic Glimmer, im gesamten Quartär kryoklastisch zerkleinert worden sein (SCHEFFER, MEYER \& GEBHARDT 1966), was auch im kalkhaltigen Tschernosem nachweisbar ist (ZHANG 1989: 113) und eine gewisse Zunahme der Grobtonfraktion bewirkt hat (Bronger 1976). Doch deutliche Bv-Horizonte wurden erst nach der Entkalkung bei saurer Bodenreaktion durch Zunahme der chemischen Silicatverwitterung gebildet.

Über die chemische Verwitterung der Alkalifeldspäte im Holozän wird unterschiedlich berichtet. Dies ist verständlich, denn neben dem Zerkleinerungsgrad hat die Versauerung der Böden einen großen Einfluss auf die Silicatverwitterung, und sie ist unterschiedlich weit fortgeschritten. Verwitterungsanfälliger sind die Glimmer, die als flache Mineralplättchen durch die eiszeitlichen Staubstürme über ganz Mitteleuropa verbreitet worden sind und in fast allen oberflächennahen Substraten eine Rolle spielen, insbesondere der Biotit. Die Biotit-Verwitterung ist der Leitprozess der rezenten Silicatverwitterung in den pleisto-

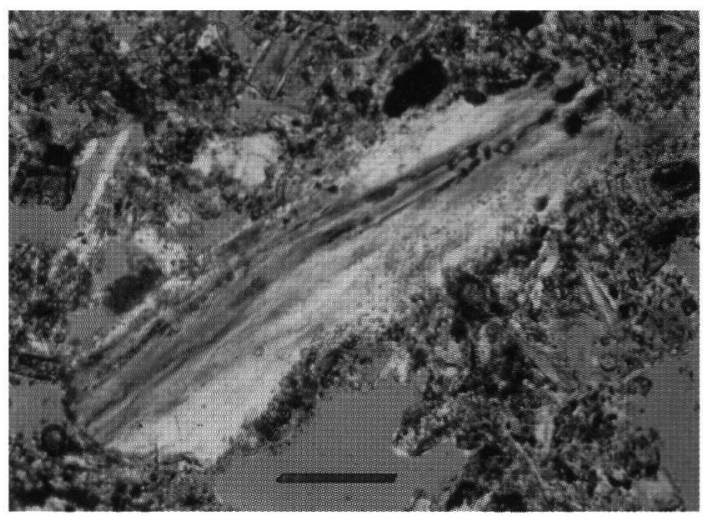

Abb.1: Verwitterndes Biotitstück. Teilweise gekreuzte Polarisatoren, Maßstab $50 \mu \mathrm{m}$.

Fig.1: Piece of mica, weathering. Partly crossed nicols, scale $50 \mu \mathrm{m}$. zänen Substraten Mitteleuropas (Schlichting \& Blume: 1961, Bronger 1966: 45, Reichenbach \& Rich 1975) (Abb.1). Der eisen- und magnesiumhaltige Biotit als Hauptquelle der Verbraunung und Verlehmung verwittert leicht zu DreischichtTonmineralen und Eisenoxid und ist daher ein guter Indikator für den Grad der Silicatverwitterung, während der resistentere Muskovit schlechter zu beurteilen ist. Bei den rezenten Braunerden aus periglaziären Lagen, bei denen eine Bilanzierung schwierig ist, zeigt das Mikroskop in Hauptund Mittellage äolisch zugeführten Glimmer, und zwar auch Biotit, soweit dieser nicht bereits verwittert ist. Über die meist gering beteiligten Schwerminerale muss hier nicht referiert werden, und die Plagioklase verwittern bei fast gleicher Gestalt wegen beachtlicher chemischer Unterschiede unterschiedlich schnell.

Der Zeitbedarf der vollständigen Biotitverwitterung, der mikroskopisch feststellbaren Umwandlung aller Biotitplättchen in Tonminerale und Eisenoxid, vergrößert sich mit wachsender Menge und Größe der Biotitplättchen. In reinen Lössen mit dominanter Fernkomponente ist nach eigenen Befunden der Biotit im oberen halben Meter der rezenten Braunerden und Lessivés Mitteleuropas weitgehend verbraucht, soweit nicht Erosion oder Staubzufuhr für eine Regradation gesorgt haben.

Das vorherrschende Tonmineral der holozänen Silicatverwitterung in Mitteleuropa, der weitgehend dioktaedrische, Al-bestimmte Illit, ist dann bei $\mathrm{pH}$-Werten über 4,5 recht stabil, für seine Weiterverwitterung nennen REICHENBACH \& RicH (1975: 82) eine Beispielzahl von 0,1\% je Jahrtau-

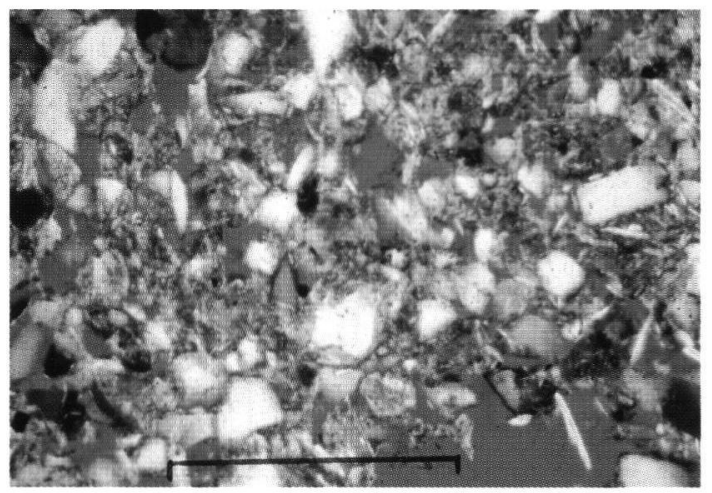

Abb.2: Tonlinsen in der Feinsubstanz (gelb), Muskovitleisten, Quarz u. a.. Teilweise gekreuzte Polarisatoren, Maßstab 0,2 mm.

Fig.2: Lenticular clay in the fine material (yellow), pieces of muskovite, quartz and others. Partly crossed nicols, scale $0.2 \mathrm{~mm}$. 
send, abgelesen am Kaliumverlust. Bei ersatzlosem Ernteentzug des Kaliums, der anthropogen bedingt ist und insofern unser Thema nicht berührt, ist die Illit-Degradierung allerdings viel höher, sie betrug z. B. beim Dauerfeldversuch „Ewiger Roggenbau“ im Oberboden einer Griserde bei Halle/Saale ca. 10\% im Jahrhundert (Reuter \& Leinweber 1989: 172); vgl. auch die Angaben bei TribuTH (1981), mit noch stärkerem K-Entzug.

Mikroskopisch ist sichtbar, dass sich in situ aus den Glimmerplättchen Tonlinsen von ca. $10 \mu \mathrm{m}$ Länge gebildet haben (Abb.2), die diffus begrenzt sind, nicht selten schon zu zusammenhängenden Gebilden verflossen sind und damit die Annahme erlauben, dass sie dispergierbar sind. Aus Muskovit gebildete Tonlinsen enthalten oft noch einen Glimmer-Kern. Eisenoxide, die bei der Verwitterung von Biotit entstanden sind, liegen lockerflockig oder als Körnchen außerhalb der Tonaggregate. Wie weit es sich dabei auch um mit Eisenoxiden umhüllte Tonteilchen handeln kann, lässt sich lichtmikroskopisch nicht feststellen.

Bei der Silicatverwitterung entsteht also unmittelbar fließfähiger, doppelbrechender Ton, das von Kubiena (z. B. 1970) geforderte Braunlehmteilplasma. Die vom verwitternden Glimmer geprägten $\mathrm{Bv}$-Horizonte mit doppelbrechenden Tonlinsen in der Matrix, die im eiszeitlich beeinflussten Gebiet weit verbreitet gewesen sein müssen, unterscheiden sich in diesem wichtigen Merkmal von anderen Bv-Horizonten. Dies lässt sich nicht makroskopisch feststellen und dann als Horizontsymbol darstellen, und leider steht in der deutschen Bodensystematik auch der Subtyp „Basenreiche Braunerde“, der im Gebiet weitgehend, wenngleich nicht ausschließlich, Bv-Horizonte mit glimmerbürtigen Tonlinsen besitzt, für die Zuordnung nicht mehr zur Verfügung.

\subsection{Tonverlagerung}

Da Entkalkung und Silicatverwitterung auch in interstadialen Böden stattgefunden haben, konzentriert sich die Diskussion weitgehend auf den Prozess, der unter geeigneten Bedingungen anschließend stattgefunden hat, die Tonverlagerung oder Lessivierung. Mikromorphologisch wurde die Tonverlagerung zunächst in Nordamerika (Frei \& Cline 1949, zitiert bei Altemüller 1962), dann aber auch in Böden Norddeutschlands nachgewiesen (Altemüller 1962, Reuter 1962).

\subsubsection{Substrat-Voraussetzungen}

Voraussetzung der Tonverlagerung ist beweglicher, hinreichend feiner Ton in der Matrix. Die
Grenzgröße der Partikel hängt von den jeweils anliegenden Poren ab. Bei verlagertem Ton herrscht die Fraktion $<0,15 \mu \mathrm{m}$ vor (Schlichting \& Blume 1961). In unverwitterten, tonarmen Substraten, insbesondere unverwittertem Löss, muss für die Lessivierung erst geeigneter Ton durch Entkalkung und Silicatverwitterung bereitgestellt werden, was einen gewissen Gehalt an Glimmer voraussetzt.

Weitere Voraussetzungen sind ein System kontinuierlicher Grobporen, in denen das Wasser die für den Tontransport hinreichende Geschwindigkeit erreichen kann, sowie gröbere Mittelporen in der Bodenmatrix. Ein zu tonreiches Material steht der Tonbewegung daher entgegen. Die Bedeutung der Korngrößenzusammensetzung für die Transportvorgänge im Boden und vor allem für die Tonverlagerung ist besonders von REUTER (1957/58) gewürdigt und mit der Unterscheidung eines stark ausgeprägten, eines mäßigen und eines unzureichenden Filtergerüstes verknüpft worden. Größere Gehalte an quellfähigem Ton stören die Tonverlagerung durch Peloturbation, die zur Rüickmischung führt. Bei der Tonverlagerung lassen sich mehrere Teilprozesse unterscheiden, die in verschiedenen Substraten eine unterschiedlich große Rolle spielen.

\subsubsection{Peptisierung und Stabilisierung}

Nach allgemeiner Überzeugung sind die Dreischicht-Tonminerale bei $\mathrm{pH}>6,5$ von Ca-Ionen und bei $\mathrm{pH}<4,5$ von Al-Ionen und schließlich auch Fe-Ionen geflockt und in der Enge der Matrix unbeweglich.

Fast alle eiszeitlich bereitgestellten Substrate waren anfangs kalkhaltig und haben dann eine Säurezufuhr erfahren. Nur in der Zeitspanne zwischen Entkalkung und stärkerer Versauerung konnten die doppelbrechenden Tonlinsen aus der Glimmerverwitterung zerfließen. War die Pufferung schlecht, wurde die Spanne von pH 6,5 bis pH 4,5 schnell durchlaufen, eventuell vorhandener doppelbrechender Ton aus der Glimmerverwitterung vererdete bei weiterer Versauerung und eine Tonverlagerung war in der Matrix nicht mehr möglich. Wohl war dann die Verwitterung noch vorhandener Silicate sogar intensiver und führte zur Bildung jüngerer, andersartiger $\mathrm{Bv}-\mathrm{Ho}-$ rizonte (REuTER 1962, 1999), in denen nur geflockte Bodenkolloide (Braunerde-Teilplasma sensu Kubiena) vorhanden sind. Auch in gut gepufferten Substraten konnte, bedingt durch Klima, Vegetation oder längere Entwicklungszeit 
(z. B. auf saalezeitlichen Moränen), hier und da die Versauerung fortschreiten und der weitgehend schon verlagerte Fließton stabilisiert werden (Fahlerdebildung).

Die starke Tonwanderung in bandkeramischen Grubenfüllungen wurde auf Pottasche-Einwirkung zurückgeführt (SLAGER \& VAN DE WETERING 1977), und auch in natürlichen Böden ist ein solcher Prozess nicht ausgeschlossen. Daher ist auch eine Ton-Dispersion nach Waldbränden durch Pottasche bei temporär alkalischer Reaktion, Aktivierung von Kieselsäure und Tonbelegung mit $\mathrm{K}^{+}$-Ionen diskutabel.

\subsubsection{Tonwanderung in der Matrix}

Ein besonders langsamer Teilprozess ist die Wanderung von Ton mit der Bodenlösung durch die Mittelporen der Matrix zu den Grobporen. Geschwindigkeit und Richtung der Wasserbewegung in der Matrix sind von den Gradienten der Wasserspannung sowie von Weite und Verlauf der Poren bestimmt, die Fließgeschwindigkeit liegt in der Größenordnung der Wasserleitfähigkeit beim entsprechenden pF-Wert. In den Mittelporen der schluffreichen Böden liegt diese unter 10-5 cm/s (Schachtschabel et al. 1998: 194, Abb. 5.4-9), so dass hier nur ein schwaches Kriechen der Tonplättchen erwartet werden kann.

Bei schluffigen Böden legen mikroskopische Bilder sogar die Vermutung nahe, dass sich während ihrer Bewegung in der Matrix die abgelösten Tonplättchen nur auf den Tonlinsen verschoben, dabei aber Kontakt behalten haben und oft $\mathrm{zu}$ größeren (in Bewegungsrichtung $>10$ bis einige $100 \mu \mathrm{m}$ ), doppelbrechenden Tongebilden zusammengelaufen sind (Abb. 3).

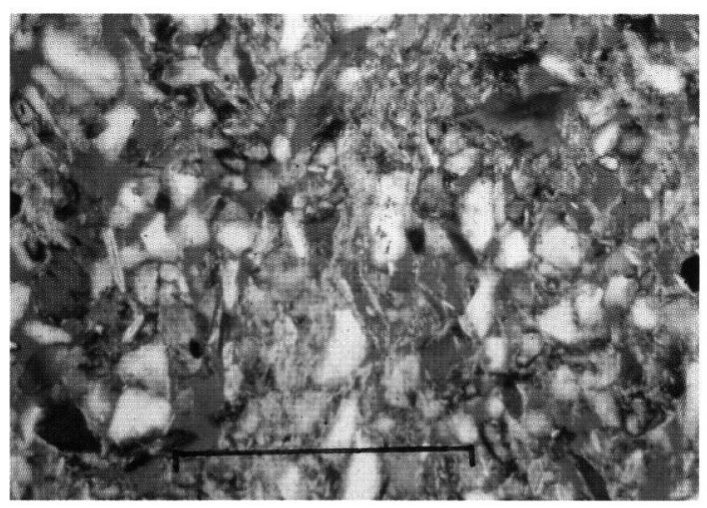

Abb.3: Gerichtet fließender Ton (gelb). Teilweise gekreuzte Polarisatoren, Maßstab 0,2 mm.

Fig.3: Directed-moving clay (yellow). Partly crossed nicols, scale $0.2 \mathrm{~mm}$
Einen Horizont, der dem späteren Al-Horizont vor oder während der Lessivierung genau entspricht, scheint es in Mitteleuropa nicht mehr zu geben; wohl ist das Zusammenfließen orientierten Tones in manchen Bv-Horizonten und schwach entwickelten Bt-Horizonten zu sehen. Da diese aber tiefer liegen, sollte das Gefüge im prospektiven Al-Horizont lockerer gewesen sein, z. B. ein schwach entwickeltes Subpolyedergefüge mit wechselnder Lokalisierung der Poren, wobei auch ein biogenes Schwammgefüge in Frage kommt. In aktiven Ah-Horizonten hätte allerdings die Bioturbation gestört.

Bei Zunahme des Tongehaltes wird der Anteil der Mittelporen immer geringer und der Weg des Tons zu den Grobporen länger. Bei einer gesättigten Leitfähigkeit unter $10^{-6} \mathrm{~cm} / \mathrm{s}$ ist ein effektiver Tontransport nicht mehr vorstellbar. Dies lässt sich wahrscheinlich nicht experimentell belegen, ist aber plausibel und wird der Grund dafür sein, dass man in sehr dichtem Material keine Tonverlagerung feststellt.

Andererseits muss jede Vergröberung des Porensystems und jede Verringerung des Abstandes zu Grobporen den Teilprozess erheblich beschleunigt haben. Wenn die feineren Korngrößenfraktionen gegenüber den gröberen zurücktreten, bleiben zwischen den gröberen Körnern zunehmend primäre Grobporen offen. Daher liegen in sand- und kiesreichem Material viele Tonpartikel unmittelbar an Grobporen, und es ist keine langsame Tonwanderung durch die Matrix in die Prozessfolge eingeschaltet.

\subsubsection{Der hohe Zeitbedarf bestätigt die angenommene Bewegungsweise}

Es wäre nun sehr wünschenswert, wenigstens an einem Beispiel die Konsequenzen der angenommenen langen Zeitspanne des Matrixtransportes für die Bewegung der Tonteilchen abzuschätzen. Die Verallgemeinerung solcher Abschätzungen ist wegen der sehr großen Vielfalt der Porensysteme allerdings kaum möglich.

Als Beispiel kann ein Lösslehm dienen, der sich zu einem Lessivé (Bodentyp Parabraunerde) entwickelt. Der Zeitbedarf für den Tontransport in der Matrix wird hier mit 500 Jahren eher niedrig angenommen. Wenn der mittlere Weg der Tonteilchen zur nächstgelegenen Wand eines kontinuierlichen Grobporensystems $5 \mathrm{~mm}$ beträgt, sind pro Jahr $0,01 \mathrm{~mm}=10 \mu \mathrm{m}$ zurückzulegen. Das ist mit der angenommenen Kriechbewegung auf einem Tonaggregat verträglich, aber nicht mit 
einem freien Transport der einzelnen Tonplättchen nach vollständiger Ablösung vom Aggregat. Die Geschwindigkeit des Bodenwassers im Boden nach Leerung der Grobporen wurde oben als $<10^{-5} \mathrm{~cm} / \mathrm{s}$ oder $<0,1 \mu \mathrm{m} / \mathrm{s}$ gekennzeichnet, woran die Festsubstanz nicht und die Feinporen praktisch nicht beteiligt sind. Bei einem Mittelporengehalt von $15 \%$ errechnet sich daraus eine Spitzengeschwindigkeit in den Mittelporen von $<0,7 \mu \mathrm{m} / \mathrm{s}$, was ebenfalls gegen einen freien Tontransport in den Mittelporen spricht.

\subsubsection{Tonwanderung in weiten Grobporen}

Der Ton, der sich an den Wänden kontinuierlicher Grobporen, sogenannter Leitbahnen, befindet (Abb.4), wird vom Sickerwasser weitergetragen, das der Gravitation folgt und entsprechend der jeweiligen Porenweite relativ schnell zu fließen vermag. Tritt Niederschlags- oder Schmelzwasser in die Grobporen ein, dann beginnt dort der Wasserfluss schon bevor die Saugspannung ins Gleichgewicht kommt. Wenn keine Blockaden (z. B. Luft) vorhanden sind, kann er anfangs sehr schnell sein und entspricht schließlich, solange der Niederschlag nicht wesentlich unter der Versickerungsrate liegt, in der Größenordnung dem gesättigten Fluss, bei dem das Wasser in Lehmböden je Stunde etwa $3 \mathrm{~cm}$ tiefer gelangt. Auch wenn das Wasser jeweils nur wenig Ton von den Wänden nimmt und nicht weit nach unten trägt, zeigt eine Betrachtung dieser Dimensionen doch, dass der hohe Zeitbedarf der Lessi-

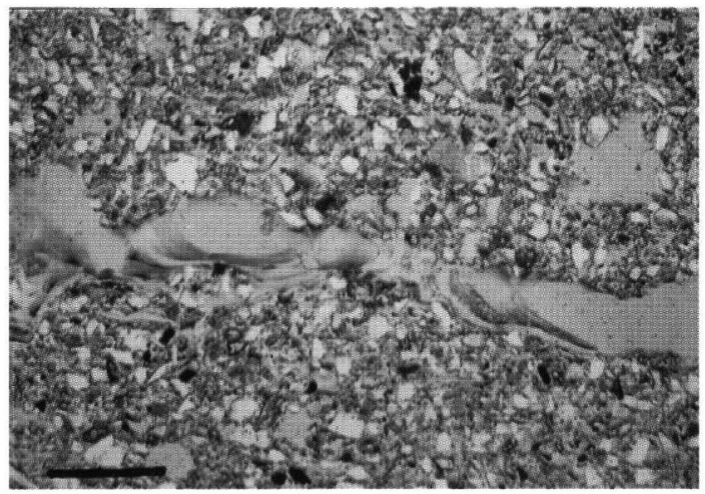

Abb.4: Tonbeläge an röhrenförmiger Leitbahn. Diese ist nicht gerade, so dass man teils ins Innere, teils auf die tonbedeckte Wand schaut. Die Röhre ist also nirgends verstopft. Teilweise gekreuzte Polarisatoren, Maßstab $0,2 \mathrm{~mm}$.

Fig. 4: Clay cutans on the wall of a tube. The tube is not straight, and either the void centre is visible, or the claycovered wall. Hence the tube is not blocked up anywhere. Partly crossed nicols, scale $0.2 \mathrm{~mm}$. vierung in Lehmen nicht mit dem Transport durch die Grobporen zusammenhängt.

Unter der Turbationszone haben die Lehmböden weitgehend kohärentes Gefüge, die Fließgeschwindigkeit wird gering, nur wenige Grobporen führen tiefer, und in den Zeiten, in denen eine hydraulische Wasserscheide ausgebildet ist (Zepp 1987: 86), ist dort die gravitative Wasserund Tonbewegung unterbrochen.

Bei hohem Anteil der groben Korngrößenfraktionen liegt etwa vorhandener Ton den Grobporen direkt an (Abb.5). Dann ist der relativ schnelle Transport in weiten Grobporen allein für die Tonwanderung maßgebend, z. B., wenn Ton aus Hochflutlehm oder Löss in Terrassenmaterial eingewandert ist, oder wenn die Silicatverwitterung in grobem Material Tonminerale produziert hat. Unter diesen Bedingungen spielt die Flockung und damit das chemische Milieu der Horizonte keine große Rolle mehr, weil kein chemischer Gleichgewichtszustand erreicht wird.

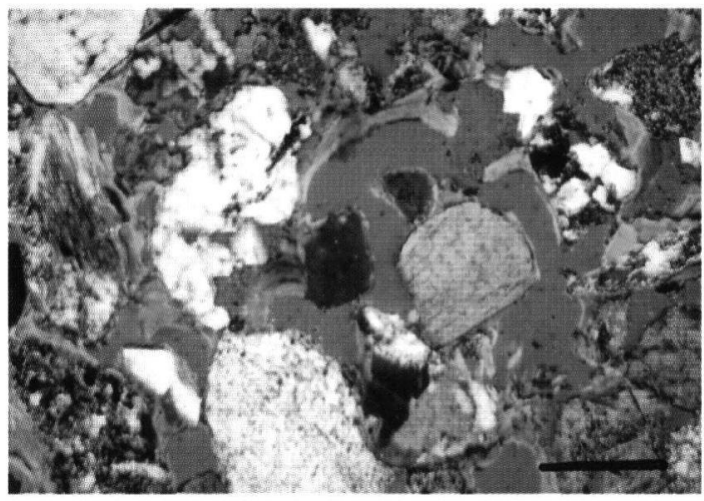

Abb.5: Fiefston in grobem Material (gelb bis rotbraun, Porenraum gleichmäßig hellbraun). Teilweise gekreuzte Polarisatoren, Maßstab 0,2 mm.

Fig.5: Mobile clay between coarse material (yellow to reddish brown, pore space uniformly light brown). Partly crossed nicols, scale $0.2 \mathrm{~mm}$.

\subsubsection{Tonverarmung im Oberboden}

Das Gegenstück zum Bt-Horizont mit Ton- und Eisenoxid-Anreicherung ist der an Ton und entsprechend an Eisen verarmte Oberboden, insbesondere der Al-Horizont. Seine Feinsubstanz ist im mikroskopischen Bild durch Mineraldetritus bestimmt, enthält nur wenig doppelbrechenden Ton, insbesondere kein Feintonplasma in Leitbahnen, erscheint heller, und das Gefüge des kolloidverarmten Materials ist relativ locker. Das sind die Eigenschaften eines Eluvialgefüges. Reste kleiner Fließton-Ansammlungen, die bei Versaue- 
rung leicht vererden, sowie Eisenoxid-Konzentrationen sind dabei nicht selten. Unter landwirtschaftlicher Nutzung ist der besonders erosionsanfällige Al-Horizont allerdings meist abgetragen, und in quartären Paläoböden wurden die A-Horizonte oft während der auf die Bodenbildung folgenden Kaltzeit aufgearbeitet.

In den weiten Grobporen breitet sich die Kolloidverarmung noch nach unten aus, wenn von oben kein Ton mehr nachfließt. Im oberen Bt-Horizont können nun statt Fließton reine Schluffkörner in den Leitbahnen auftreten. KundLLer (1961) beschreibt dies allerdings für die gröber texturierte Grundmoräne, was bei einer zeitlichen Zuordnung berücksichtigt werden muss.

\subsubsection{Verlagerung von schwach sortiertem Mate-} rial und die Unsicherheit des makroskopischen Nachweises der Tonverlagerung

Die Verlagerung schlecht sortierten Materials, das meist von der Bodenoberfläche abgelöst wird und unmittelbar in das Grobporensystem gelangt, wo es allein oder im Wechsel mit reinem Ton Beläge bildet, ist eine verbreitete Erscheinung (KEMP 1999). Es handelt sich um einen schnellen Prozess, der in einzelnen Schüben (episodisch) abläuft. In versauerten Lessivés mit Ap-Al-Bt-IIBtIIIBbt-IIIC-Profil aus schluffreichen periglaziären Lagen über sandigem Material in NW-Frankreich und Großbritannien wechseln in den Leitbahnen reine, durch Tonwanderung entstandene Tonbeläge mit inhomogenen Belägen aus horizonteigenem Material konzentrisch ab (CurMi 1987). In Material, dessen Textur gröber als Löss ist, ist der mikroskopische Nachweis reiner Tonbeläge für den Nachweis eines Interglazialbodens wahrscheinlich unverzichtbar.

Bei makroskopischen Profiluntersuchungen werden gewöhnlich Tonbeläge auf den Flächen der Absonderungsgefüge als Ergebnis der Tonverlagerung gewertet. Aber makroskopisch sichtbare, schwächere Tonwandbeläge entsprechen mikromorphologisch oft nur einer Tonorientierung, während sich eine Tonverlagerung an doppelbrechendem Feintonplasma besonders in den Leitbahnen zeigt (BRONGER 1976: 212). Die Verwechslung des durch Scherbewegungen orientierten, matrixeigenen Tons mit illuviiertem Ton ist ein häufiger Fehler bei der makroskopischen Untersuchung. Andererseits zeigt das Mikroskop auf Gefügeflächen nicht immer Fließtonbeläge, wenn diese in Röhren in Bt-Horizonten nachzuweisen sind. Frische Tonbeläge in Wurzelröhren können ohne Unterbrechung durch mehrere PolyederGrenzflächen hindurchziehen (KundLER 1961). Bei Regen füllt das Wasser die Risse von unten nach oben, die Segregate quellen und die Risse schließen sich. Röhren aber bleiben bis zur eventuellen Zuschlämmung offen. Für stratigraphische Untersuchungen kann es sinnvoll sein, nicht nur diese Formen zu unterscheiden, sondern noch weiter zu differenzieren. So weisen Tonbeläge in abgeschlossenen Röhren innerhalb von Aggregaten auf eine ältere Lessivierungsphase hin (Kemp et al. 1998).

Besonders in Spalten findet man oft schwach sortiertes, direkt eingespültes Oberflächenmaterial. Obwohl dieses keine Tonverlagerung anzeigt, kann es makroskopisch mit Fließton verwechselt werden, wenn es beim Spaltenschluss geglättet wurde. Hier ist eine mikroskopische Klärung empfehlenswert und auch erfolgversprechend.

\section{Bildungsbedingungen für Bt-Horizonte, deren Entwicklung nicht unbedingt ein Interglazial benötigt}

Diese Betrachtung der verschiedenen Varianten der Bodenentwicklung bis zur Lessivierung führt zu dem Schluss, dass der Zeitbedarf sehr unterschiedlich ist. Unter bestimmten Bedingungen kann die Tonverlagerung relativ schnell ablaufen, mitunter innerhalb weniger Jahre (STEPHAN 1993: 82). Diese Bedingungen sind nun zusammenzustellen; denn ihnen zugeordnete Bodenhorizonte zeigen keine sicheren Interglazialböden an. Ferner ist zu diskutieren, wann bei umgelagerten Böden die bodenbildenden Prozesse vor und nach der Umlagerung zusammengefasst werden können. Dann erst wissen wir, welche Formen der Lessivierung ein Interglazial erfordern.

\subsection{Beschleunigung der Bildung von Bt-Horizonten}

Kalk-oder Silicatmangel

Kalkmangel im Ausgangsmaterial bedeutet eine erhebliche Beschleunigung der Bodenentwicklung, da dann die für eine Entkalkung notwendige Zeit sehr kurz ist oder wegfällt. In schluffreichen Substraten mit langsamer innerer Dränung und, um eine ungefähre Zahl zu nennen, mit über $5 \%$ Glimmer braucht die Silicatverwitterung viel Zeit. Dagegen verwittert ein mageres, glimmerarmes Substrat schneller durch, gibt allerdings wenig Ton. Es durchläuft auch die pH-Spanne der Lessivierung schnell, so dass mengenmäßig nur 
wenig Ton verlagert wird, was dennoch in Prozent des Tongehaltes erheblich sein kann. Geringer Tongehalt des Substrates und geringe absolute Tondifferenz zwischen Bt-Horizont und Substrat gebieten also eine zurückhaltende Beurteilung.

Vorverwitterung und Vorlessivierung umgelagerten Bodenmaterials

Bei vorverwittertem allochthonem Material ist die Zeit für Entkalkung und Silicatverwitterung weitgehend schon an einem hangaufwärts gelegenen Ort abgelaufen. Bei sekundär aufgekalktem Material erfordert die Untersuchung große Sorgfalt, da sich nicht nur die Farbe, sondern auch das Gefühl bei der Fingerprobe mit der Entkalkung erheblich ändert. Die Verbraunung durch Entkalkung spielt besonders beim kalkhaltigen Braunauenboden (Kalkhaltige Vega) eine Rolle (Stephan 1993), bei dessen Entkalkung sich der Tongehalt nur unwesentlich verändert.

Zum Nachweis autochthoner Tonverlagerung gehören ungestörte Tonbeläge auf Porenwänden. Allochthones Bt-Material kann mikroskopisch an zerbrochenen, in die Bodenmatrix aufgenommenen Tonbelägen erkannt werden, die auch nach Verlagerung über längere Strecken noch gut erkennbar sind (Abb. 6). Ein Problem bietet die Unterscheidung von pedoturbat gestörtem, parautochthonem und allochthonem Material. Bei der Verlagerung werden jedenfalls größere und unregelmäßige Fragmente mit wachsender Intensität der Störung zunehmend zerteilt und zugerundet. Sind intakte neben fragmentierten Tonbelägen vorhanden, so müssen die intakten

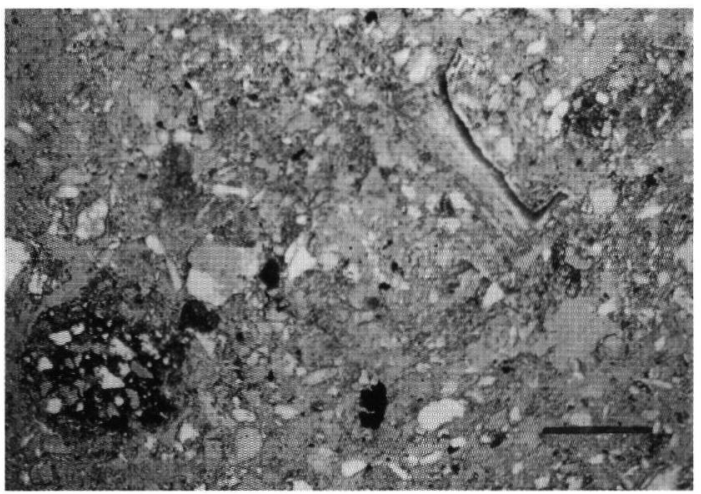

Abb.6: Bodensediment mit Tonbelags-Fragment (gelb mit braunem Streifen) in der Matrix. Teilweise gekreuzte Polarisatoren, Maßstab 0,2 $\mathrm{mm}$.

Fig.6: Soil sediment with fragment of a clay cutan (yellow with brown stripe) in the soil-matrix. Partly crossed nicols, scale $0.2 \mathrm{~mm}$. nicht aus fein verteiltem Ton neu entstanden sein, sondern sie können sich auch durch Remobilisierung von Tonbelags-Resten gebildet haben, und das kann eine Zeiteinsparung bei der Weiterentwicklung bedeuten.

Verlagertes Bt-Material kann nur dann ein Interglazial sicher anzeigen, wenn Vorverwitterung und Tonwanderung nicht durch eine Kaltzeit getrennt sind. Man muss also z. B. sicherstellen, dass sich der rezente Bt-Horizont nicht in verlagertem Eemboden-Material gebildet hat.

Beispiele für schnelle Tonverlagerung in umgelagertem Material geben hallstattzeitliche (ca. $600 \mathrm{v}$ Chr.) Grabhügel im Taunus (FICKEL et al. 1977, Beckmann et al. 1978). Das kalkfreie Material ist vorverwittert, enthält reaktivierbare Relikte von Tonbelägen aus einem ehemaligen Bt-Horizont, aber auch viel porenreiches Eluvialgefüge, der absolute Tongehalt ist gering, und es war beim Aufschütten gelockert worden. Dies ist eine die Entwicklung beschleunigende, anthropogen verursachte Ausnahmesituation.

Ein sehr verbreitetes Substrat ist das Kolluvium, das aber, als Folge des Ackerbaues, an das Holozän gebunden ist. Es handelt sich um das bei mangelnder Vegetationsbedeckung von den Hängen abgespülte, durch Anteile der Ap-Horizonte etwas humose Material, sowcit cs tiefer gelegene Hangbereiche bedeckt. Aus Löss- oder Geschiebemergel-Böden entstandene Kolluvien der Bronze- und Eisenzeit sind weitgehend während der Völkerwanderungszeit, solche des Mittelalters während anschließender Bewaldungsphasen lessiviert worden (BORK et al. 1998). Dies erscheint zwar als zeitverkürzte Bildung; aber hier ist wiederum die gesamte Entwicklungszeit, auch die am Oberhang abgelaufene, relevant, und diese reicht bis zum beginnenden Holozän oder sogar zu den spätglazialen Interstadialen zurück.

\section{Sand- und Kies-Dominanz}

Im Abschnitt 3.3 .5 wurde dargestellt, dass die Tonverlagerung in sand- und kiesreichen Horizonten sehr schnell ablaufen kann. Dann können Bt-Horizonte nicht als Zeichen für ein Interglazial gewertet werden, auch wenn sie ungewöhnlich mächtig entwickelt sind.

Bei Dominanz der Korngrößen $>63 \mu \mathrm{m}$ benötigte die Bildung von Bt-Horizonten im Geschiebemergel Norddeutschlands kein Interglazial. Hier kann die Tonverlagerung also nicht zur zeitlichen 
Einordnung herangezogen werden. Ebenso wenig müssen die 2-3 m mächtigen Bt- und Bvt-Horizonte an der Oberfläche der oberen Hochterrasse aus dem Mittelriss (Bıbus \& Kösel 1997: 60) zwingend als Interglazialbildungen gewertet werden, wenn der eingeschlämmte Ton aus dem hangenden Auenlehm stammt. Interessant ist, dass hier die Tonwanderung durch den Einfluss der Carbonate nicht verhindert worden ist, was wohl daran liegt, dass sich beim schnellen Transport kein chemisches Gleichgewicht einstellen konnte.

Die verschiedenen Prozesse und ihre Bedeutung für die Tonverlagerung sind in Tab. 1 nochmals zusammengestellt.

\subsection{Weitere für die Beurteilung kritische Bodenbildungsverhältnisse}

Verdoppelung und Verstärkung von Böden durch Verlagerung

Nicht nur die Umlagerung verwitterten und dann

Tab. 1: Kennzeichnung der Prozesse und ihrer Bedeutung für die Tonverlagerung

Tab.1: Characterization of the processes and their significance for the clay illuviation

\begin{tabular}{|c|c|c|c|}
\hline PROZESS & $\begin{array}{l}\text { zeitliche } \\
\text { Einschränkungen }\end{array}$ & Kennzeichnung & wirkt auf/ist Voraussetzung für \\
\hline SUBSTRATBILDUNG & $\begin{array}{l}\text { vorwiegend } \\
\text { kaltzeitlich }\end{array}$ & $\begin{array}{l}\text { Kryoklastik, } \\
\text { äolischer Eintrag, } \\
\text { Kryoturbation und Solifluktion }\end{array}$ & $\begin{array}{l}\text { Korngrößenspektrum und damit } \\
\text { Porensystem, Gehalt an Kalk und } \\
\text { Glimmer (insbes. Biotit) }\end{array}$ \\
\hline ENTKALKUNG & $\begin{array}{l}\text { vorwiegend } \\
\text { warmzeitlich }\end{array}$ & $\begin{array}{l}\text { Kalklösung durch Säuren, } \\
\text { vorwiegend durch Kohlensäure }\end{array}$ & $\begin{array}{l}\text { erlaubt effiziente Silicatverwitte- } \\
\text { rung und Ton-Dispergierung }\end{array}$ \\
\hline $\begin{array}{l}\text { CHEMISCHE } \\
\text { SILICAT- } \\
\text { VERWITTERUNG }\end{array}$ & warmzeitlich & $\begin{array}{l}\text { nach Entkalkung, } \\
\text { durch Hydrolyse, Protolyse und } \\
\text { Oxidation, zunächst aus Glimmer }\end{array}$ & $\begin{array}{l}\text { erzeugt vorwiegend unmittelbar } \\
\text { flieisfähige Dreischicht-Ton- } \\
\text { minerale }\end{array}$ \\
\hline
\end{tabular}

\section{TONVERLAGERUNG}

Dispergierung durch Wasser nach teilweisem

Ersatz der mehrwertigen

Kationen zwischen $\mathrm{pH} 6,5$

und 4,5; Dispergierung durch

$\mathrm{K}_{2} \mathrm{CO}_{3}$ möglich? strenge Voraussetzung der

Tonbewegung in der Matrix unter Dispersionsbedingungen erlaubt Fortsetzung der Lessivierung

verlagerter

Tonbelagsfragmente

Transport durch -

die Matrix
Transport im

Grobporen-System
Bewegung mit Bodenwasser in Mittelporen, extrem langsam (Jahrhunderte bis Jahrtausende)

schnellere Bewegung nach unten (Jahre bis Jahrhunderte je nach Wassermenge und Porensystem)

INHOMOGENE

EINSCHLÄMMUNG

FLOCKUNG

RÜCKMISCHUNG episodische Ein- und Durchschlämmung von Schluff, Ton und ggf. Humus in Grobporen

durch Al- und Fe-Ionen bei $\mathrm{pH}<4,5$ bzw. $<3,5$; durch Ca-Ionen im Cc-Horizont

durch Pedoturbationen sowie kaltzeitliche Prozesse, Tonbelagsfragmente teils erhalten für Ton aus dem Inneren der Matrix Voraussetzung des Transports in Grobporen

verursacht die Ausbildung von Bt-Horizonten

hat keine direkte Beziehung zur Tonwanderung; kann mit dieser alternieren

ist abhängig vom Erreichen des chemischen Gleichgewichtes, beendet die Tonbewegung

wirkt der Tonverlagerung entgegen unterbricht Prozesskette, zerstört

Profil-Differenzierung; Ton-

belagsfragmente teils erhalten
BEI BEWERTUNG VON

Bt-HORIZONTEN

BERÜCKSICHTIGEN! 
meist schon lessivierten Materials muss aufgedeckt werden, weil dann nur für die summierte Zeit Aussagen möglich sind. Vielmehr kann auch die Überdeckung von Böden mit transportiertem Material Warmzeiten vortäuschen, wenn auch in diesem Material noch während derselben Phase ein Boden gebildet wurde. Die Zahl der Interglaziale oder Interstadiale kann überschätzt werden, wenn nicht zwischen Löss in situ und durch Paläoböden getrenntem umgelagertem Löss unterschieden wird (Mücher 1986: 16). Auch ohne trennenden Paläoboden kann allochthones Bodenmaterial eine zu starke Bodenbildung vortäuschen, wenn transportbedingte Merkmale wie verlagerte Tonbelagsrelikte oder verlagerte FeMn-Konkretionen unbeachtet bleiben.

Häufiger aber bleiben Interglazialböden unberücksichtigt. Oft sind sie in den verfügbaren Aufschlüssen vollkommen abgetragen. Besonders starke Bt-Horizonte können jedoch auch, wie wiederholt gezeigt wurde, durch geringe oder fehlende Sedimentation zwischen zwei Interglazialen oder zwischenzeitliche Erosion entstanden sein, indem der untere Boden in die neue Bodenbildung einbezogen wurde. Solche Bodenkomplexe sind schwer zu trennen (Beispiel Lössaufschluss Reinheim/Odenwald bei Semmer 1974). Das Einbeziehen des Eembodens in die holozäne Bodenbildung lässt sich oft durch ein lumineszenzdatiertes weichselzeitliches Alter des Liegenden ausschließen, aber bei älteren Böden werden solche Datierungen zunehmend unsicher.

Nach unten durchgreifende Prozesse

Ein schwach pufferndes, hinreichend poröses, nicht zu mächtiges Zwischensubstrat erlaubt den Durchgriff jüngerer Verwitterung und Lessivierung in ältere Substrate oder Böden. Dann kann die Bodenbildung auf dem älteren Substrat teilweise der Bodenbildung über dem Zwischensubstrat entsprechen.

Ein bekanntes Beispiel bietet das Neuwieder Becken, wo zwischen Löss der Jüngeren Tundrenzeit und älterem Weichsel-Löss mit dem Alleröd-Boden die Laacher-See-Tephra liegt. Unter der Tephra ist meist eine Pararendzina ausgebildet, manchmal aber zeigt sich Verbraunung und mitunter schwache Tonverlagerung, die mikromorphologisch durch AltemüLler nachgewiesen wurde (SCHÖNHALS 1960). Den einzigen voll entwickelten Bt-Horizont findet SCHÖNHALs allerdings in Sandlöss, wo er durch die gröbere Bodenart begünstigt wird. Als Ursache der Lessivierung wird ein Durchgriff der holozänen und damit ei- ner interglaziären Pedogenese angesehen ( $\mathrm{ROH}-$ Denburg \& Meyer 1968, Kurtenacker \& SChröder 1987, Ikinger 1996, Schirmer 1996 u.a.). Ton aus hangendem Material oder aus der Tephra selbst ist schon wegen der zahlreichen primären Grobporen in der Tephra beweglich und kann in den liegenden Boden gelangt sein. Die beiden Tonherkünfte lassen sich mikroskopisch unterscheiden (Poetsch \& Altemüller 1994).

Tonverlagerung unter Siedlungsflächen

Bei der Beschreibung der Lebensumstände der ehemaligen Bewohner archäologischer Fundplätze spielt der Boden als Archiv für die Umweltfaktoren eine zunehmende Rolle. Bei der Bodenentwicklung unter der unmittelbaren Siedlungsfläche oder in Grubenfüllungen ist aber Vorsicht geboten. Insbesondere die dort allgegenwärtige Asche verändert das pedochemische Milieu und begünstigt die Tonverlagerung (SLAGER \& VAN DE Wetering 1977, Courty \& Goldberg \& Macphail 1989: 113).

\section{Bt-Horizonte mit zweifelsfreier interglazialer Entstehung}

Wenn sie überhaupt auftreten, sind also autochthone Bt-Horizonte aus ehemals kalkhaltigen, schluffreichen Substraten, insbesondere aus Löss, sowie Bt-Horizonte oder Bt-Material in Bodensedimenten aus solchen Substraten, die während derselben Phase (dem Interglazial) vorverwittert, umgelagert und der Tonverlagerung unterworfen waren, sichere Zeichen für Interglaziale. Durchgriff der Genese von weiter oben und Asche-Einfluss unter Siedlungsflächen müssen aber ausgeschlossen sein. Treten in einem „Interstadial“ BtHorizonte auf, die den genannten strengen Maßstäben genügen, liegt wahrscheinlich ein Interglazial im eingangs festgelegten Sinne vor, was die Pollenanalyse erweisen müsste.

\section{Zum Ausbleiben der Tonverlagerung in Interglazialböden}

Abschließend ist zu betonen, dass die genannten Bedingungen für die Zugehörigkeit eines Bt-Horizontes zu einem Interglazial hinreichen, aber nicht notwendig sind. Dies ist selbstverständlich, bilden sich doch die Lessivés nur auf bestimmten Substraten in bestimmten Klimaten. So hat sich der Tschernosem in Trockengebieten (z. B. Mainzer Becken) nicht zum Lessivé weiterentwickelt, weil Silicatverwitterung und Perkolation zu gering sind, die Flockung durch Ca-Ionen und die 
Rückmischung durch Bodentiere und Bodenbearbeitung aber zu groß. Andererseits haben die Braunerden aus lössreichen über schuttdominierten periglaziären Lagen im Rheinischen Schiefergebirge trotz hinreichender Glimmerverwitterung keine Lessivierung erlitten, weil durch die schnelle Versauerung freigesetzte Al-Ionen die Dispersion des Tons verhindert haben. Schließlich wirkt auch eine hohe biologische Aktivität der Tonwanderung entgegen.

\section{Dank}

Für hilfreiche Kritik dankt der Autor den Herren Prof. Dr. Arvt Bronger, Universität Kiel, Prof. Dr. Peter Felix-Henningsen, Universität Gießen, Prof. Dr. Gerhard Reuter, Universität Rostock, sowie seiner Frau Barbara Stephan.

\section{Schriftenverzeichnis}

Altemüller, H.-J. (1962): Beitrag zur mikromorphologischen Differenzierung von durchschlämmter Parabraunerde, Podsol-Braunerde und Humus-Podsol. - Z. Pflanzenernähr., Düng., Bodenkd., 98: 247-258, 8 Abb.; Weinheim.

Beckmann, H., Fickel, W., Plass, W., Schrader, L., StePHAN, S., WifchmaNN, H. \& ZAKOSEK, H. (1978): Aufbau und jungholozäne Tonverlagerung in Hügelgräbern im Taunus. - Geol. Jb. Hessen, 106: 383-407, 2 Abb., 10 Tab., 3 Taf.; Wiesbaden.

Bibus, E. \& Kösel, M. (1997): Paläoböden und periglaziale Deckschichten im Rheingletschergebiet von Oberschwaben und ihre Bedeutung für Stratigraphie, Reliefentwicklung und Standort. - Tübinger geowiss. Arb. (TGA), Reihe D, 3: 91 S., 28 Abb., 6 Tab.; Tübingen.

Bork, H. R., Bork, H., Dalchow, C., Faust, B., Piorr, H.P. \& Schatz, T. (1998): Landschaftsentwicklung in Mitteleuropa. Wirkungen des Menschen auf Landschaften. - 328 S., 68 Abb., 36 Tab.; Gotha \& Stuttgart (Klett-Perthes).

Bronger, A. (1966): Lösse, ihre Verbraunungszonen und fossilen Böden. - Schriften geogr. Inst. Universität Kiel, 24: 113 S.; Kiel.

- (1976): Zur quartären Klima- und Landschaftsentwicklung des Karpatenbeckens auf (paläo-)pedologischer Grundlage. - 268 S., 10 Tab., 13 Abb., 6 Taf., Anlagen; Kiel (Geogr. Inst. Uni.).

- (1999): Löß-Paläoboden-Sequenzen Zentralasiens als Indikatoren einer globalen Klimageschichte des Quartärs? - Eiszeitalter und Gegenwart, 49: 35-54, 6 Abb.; Hannover.

Courty, M. A., Goldberg, P. \& Macphail, R. (1989): Soils and micromorphology in archaeology. - 344 S., 18 Tab., 101 Fig., 8 Taf,; Cambridge (Cambridge Univ. Press).

Curmi, P. (1987): Sur la signification des revetements complexes argileux et limoneux dans les sols lessives acides. - In: Fedoroff, N., Bresson, L. M. \& CourTY, M. A. [eds.]: Micromorphologie des Sols - Soil
Micromorphology: 251-255, 6 Abb.; Plaisir, Frankreich (Assoc. Française pour l'étude du sol).

Fickel, W., Beckmann, H., Plass, W., Schrader, L., Stephan, S., Wiechmann, H. \& ZaKosek, H. (1977): Über Bodenentwicklungen in Hügelgräbern im Taunus. Mitt. dt. bodenkundl. Ges., 25: 639-644, 1 Abb., 2 Tab.; Göttingen.

Frei \& CuIne (1949): Profile studies of normal soils of New York. II. Micromorphological studies of the graybrown podzolic-brown podzolic soil sequence. - Soil Sci., 68: 333-344, 16 Fig, 2 Tab.; Baltimore, Md.

Graedel, T. E. \& Crutzen, P. J. (1994): Chemie der Atmosphäre: Bedeutung für Klima und Umwelt. - 511 S., 222 Fig., 25 Tab.; Heidelberg (Spektrum Akad. Verl.).

IkINGER, A. (1996): Bodentypen unter Laacher See-Tephra im Mittelrheinischen Becken und ihre Deutung. - Mainzer geowiss. Mitt., 25: 223-284, 16 Abb., 6 Tab., 2 Ktn.; Mainz.

Kemp, R. A. (1999): Micromorphology of loess-paleosol sequences: a record of paleoenvironmental change. - Catena, 35: 179-197, 8 Fig.; Amsterdam.

- McDaniel, P. A. \& Busacca, A. J.. (1998): Genesis and relationship of macromorphology and micromorphology to contemporary hydrological conditions of a welded Argixeroll from Palouse in Idaho. Geoderma, 83: 309-329, 7 Fig., 2 Tab.; Amsterdam.

Kopp, E. (1965): Über Vorkommen „degradierter Steppenböden“ in den Lößgebieten das Niederrheins und Westfalens und ihre Bedeutung für die Paläobodenkunde und Bodengenese. - Eiszeitalter und Gegenwart, 16: 97-112; Öhringen, Württ.

Kublena, W. L. (1970): Micromorphological features of soil geography. - 256 S., 18 Fig., 8 Diagramm-Tafeln, 15 Farbtafeln; New Brunswick/N. J. (Rutgers Univ. Press).

Kundler, P. (1961): Lessivés (Parabraunerden, Fahlerden) aus Geschiebemergel der Würm-Eiszeit im norddeutschen Tiefland. - Z. Pflanzenernähr., Düng., Bodenkd., 95: 97-110, 7 Abb., 5 Tab; Weinheim.

Kurtenacker, M. \& Schröder, D. (1987): Eigenschaften und Genese fossiler Lößböden unter Pyroklastika des Laacher See-Gebietes. - Mitt. dt. bodenkundl. Ges. 55 (2): 785-790, 2 Tab.; Göttingen.

Mücher, H. J. (1986): Aspects of loess and loess-derived slope deposits: an experimental and micromorphological approach. - Nederlandse Geografische Studies, 23: 267 S., 40 Fig., 3 Tab.; Amsterdam.

Poetsch, T. \& Altemüller, H.-J. (1994): Mineralogische und mikromorphologische Merkmale von Böden aus Laacher See Pyroklastika. - Mitt. dt. bodenkundl. Ges., 74: 387-390; Oldenburg.

Reichenbach, Graf H. \& Rich, C. I. (1975): Fine-grained micas in soils. - In: Gieseking, J. E. [ed.]: Soil Components, 2: 60-95, 7 Fig., 3 Tab.; Heidelberg (Springer).

Reuter, G. (1957/58): Bodentypen in Mecklenburg. Wiss. Z. Uni. Rostock, 7: 663-671, 12 Abb.; Rostock.

- (1962): Lessivé-Braunerde-Interferenzen auf Geschiebemergel. - Z. Pflanzenernähr. Bodenkde., 98 : 240-246, 1 Farbtafel; Weinheim. 
- (1964): Zur Mikromorphologie lessivierter Böden in verschiedenen Klimagebieten. - In: A. JONGERIUS [ed.]: Soil Micromorphology: 213-218, 2 Abb.; Amsterdam (Elsevier).

- (1999): Statement zur Lessivé-Problematik in Mitteleuropa. - Mitt. dt. bodenkundl. Ges., 91: 1065-1068; Oldenburg.

Reuter, G. \& Leinweber, P. (1989): Bodenmineralogische Untersuchungen am Dauerfeldversuch 'Ewiger Roggenbau' zu Halle/Saale. - Kongr. u. Tag.Ber. MLU Halle-Wittenberg WB, 55: 169-175.

Rohdenburg, H. \& Meyer, B. (1968): Zur Datierung und Bodengeschichte mitteleuropäischer Oberflächenböden (Schwarzerde, Parabraunerde, Kalksteinbraunlehm): Spätglazial oder Holozän? - Göttinger bodenkdl. Ber., 6: 127-212, 12 Abb.; Göttingen.

Schachtschabel, P., Blume, H.-P., Brümmer, G., Hartge, K. H. \& SChWERTMANN, U. (1998): SchefFER/SCHACHTSCHABEL Lehrbuch der Bodenkunde. - 14. Aufl.: 494 S., 248 Abb., 100 Tab., 1 Taf.; Stuttgart (Enke).

Scheffer, F., Meyer, B. \& Gebhardt, H. (1966): Pedochemische und kryoklastische Verlehmung (Tonbildung) in Böden aus kalkreichen Lockersedimenten. - Z. Pflanzenernähr., Düng., Bodenkd., 114: 77-89; Weinheim.

ScHIRMER, W. (1996): Spätglaziale Böden unter Laacher See-Tephra.- In: Böden als Zeugen der Landschaftsentwicklung: 49-58, 1 Tab., 3 Abb.; Kiel (Landesamt für Natur und Umwelt des Landes SchleswigHolstein).

Schlichting, E. \& Blume, H.-P. (1961): Art und Ausmaß der Veränderungen des Tonmineralbstandes typischer Böden aus jungpleistozänem Geschiebemergel und ihrer Horizonte. - Z. Pflanzenernähr., Düng., Bodenkd., 95: 227-239, 4 Abb., 4 Tab.; Weinheim.

SchÖNHALS, E. (1960): Spät- und nacheiszeitliche Entwicklungsstadien von Böden aus äolischen Sedi- menten in Westdeutschland. - 7th Internat. Congr. Soil Sci., Madison, U.S.A., 4: 283-290, 1 Tab.

Semmel, A. (1974): Der Stand der Eiszeitforschung im Rhein-Main-Gebiet. - In: Semmel, A. [Hrsg.]: Das Eiszeitalter im Rhéin-Main-Gebiet. - Rhein-Main. Forsch., 78: 215 S., 21 Abb.; Frankfurt a. M.

Slager, S. \& van de Wetering, H. T. J. (1977): Soil formation in archaeological pits and adjacent loess soils in Southern Germany. - J. Archaeol. Sci., 4: 259-267, 2 Tab., 1 Fig.; London.

Stephan, S. (1993): Mikromorphologie und Genese von Böden auf den Niederterrassen des Niederrheingebietes und der Kölner Bucht. - Eiszeitalter und Gegenwart, 43: 67-86, 2 Abb., 8 Tab., 3 Taf.; Hannover.

- (1994): Interpretation von Bodendünnschliffen für die Bodengeschichte in Mitteleuropa. - Mitt. dt. bodenkundl. Ges., 74: 403-406; Oldenburg.

- (1995): Mikromorphologie und Kalkdynamik im Tschernosem Rheinhessens, - Z. Pflanzenernähr. Bodenk., 158; 187-190, 1 Tab.; Weinheim.

Tributh, H. (1981): Erste Ergebnisse über die Reduzierung der Illitgehalte durch Kaliumentzug der Pflanzen. - Mitt. dt. bodenkundl. Ges., 32: 827-834., 2 Abb., 1 Tab.; Göttingen.

Zepp, H. (1987): Wasserhaushalt und Verlagerung wasserlöslicher Stoffe in Lößdecken des Main-TaunusVorlandes. - Arbeiten zur rheinischen Landeskunde, 56: 167 S., 97 Abb.,15 Tab, 1 Beilage; Bonn.

ZHANG, T. (1989): Vergleichende Untersuchungen an deutschen und chinesischen Steppenböden. Gießener Abh. z. Agrar- u. Wirtschaftsforsch. d. europ. Ostens, 167: 174 S., 16 Abb., 15 Tab.; Berlin.

Manuskript eingegangen am 12. 11. 1999, zum Druck angenommen am 02. 08. 2000 